\title{
BIM-BASED LCA THROUGHOUT THE DESIGN PROCESS: A DYNAMIC APPROACH
}

\author{
GIANLUCA GENOVA \\ Digital Innovation Laboratory, Basler and Hoffman, Switzerland
}

\begin{abstract}
As design projects become more and more sophisticated, it becomes increasingly significant for the designers to consider environmental aspects when selecting building materials. A promising solution to tackle this challenge is an integration of Life Cycle Assessment (LCA) data into Building Information Modelling (BIM) platforms. The implementation can support the spreading of sustainability assessment to make informed choices of construction materials during the building design phase. This paper shows how a real-time environmental assessment method can be implemented to all design phases by integrating a BIM-based approach with known LCA methodologies. Mainly two environmental assessment methods are investigated and compared. The first method is called the static approach, where the LCA is done with known methods at the end of each milestone of the project. A second method is a dynamic approach where the environmental assessment is done continuously during the planning process. Several possible LCA workflows in a BIM environment with the help of the Revit as a BIM author tool and Dynamo as an integrated parametric solution are shown. The idea is to link building objects and their material specifications with national LCA databases to be able to calculate the embodied energy directly from the building information model. Taking all the data from the information model and LCA databases reduces the effort of the repetitive data input into LCA tools. The streamlined LCA methodology allows building professionals to use LCA efficiently in their daily work. Finally, this work suggests a workflow of integrating LCA with BIM for the decision-making process during the design phase to boost sustainable development in the construction industry.

Keywords: BIM-LCA integration, dynamic LCA, parametric LCA, data integration, LCA tool.
\end{abstract}

\section{INTRODUCTION}

Environmental sustainability concept encourages some essential changes in a built environment to reduce the consumption of energy and natural resources. However, the construction industry, compared to other industries, has been criticised for being a significant carbon emitter and a relatively unregulated discipline regarding control and management of environmental impact [1].

The design phase of a project has a high potential for adding value to the project regarding the reduction of the environmental impact. The initial phases have great potential for implementing changes, studying different alternatives, improving performance and reducing costs [2]. Therefore, the design phase should be considered as the first and most crucial stage for achieving sustainability. The British Government stated: "Good design is synonymous with sustainable construction" [3]. Building professionals use the Life Cycle Assessment (LCA) methodology in order to estimate the environmental impact of the building in the design stage. At this point, seeing LCA as a decision-making tool would be the right approach to sustainable project development [4].

Environmental considerations need to be integrated with the choice of building materials. If the key to sustainability is to use LCA as a design-support tool, the next step would be then to evaluate how LCA can be used effectively to influence the design choices. An encouraging possibility is an integration of building systems' LCA data into Building Information Modelling (BIM) platforms. Such implementation can support the spreading of environmental assessment and conscious choice of construction materials during the building 
design phases [5]. Therefore, BIM-integrated LCA approaches could be applied in the early phases to influence the decision-making process.

\section{OVERVIEW OF LCA METHODOLOGIES}

In this study, all known LCA-BIM integrated environmental sustainability assessments are classified into three principal methodologies.

\subsection{Conventional LCA methodology}

The most commonly used environmental impact assessment is conceptually summarised as the conventional LCA methodology, which is presented in Fig. 1. For every project, there is a source data, which can be an advanced BIM model, a 3D model or a 2D drawing. In any case, there is the need for material take-off from the project primary source data, which in the best case, is a BIM model. The take-off can be automatically generated from the primary source data if it is hosted in a parametric software like Revit or ArchiCAD [6]. However, if the project is based on 2D drawings, this material take-off has to be done through a manual calculation, which can be challenging.

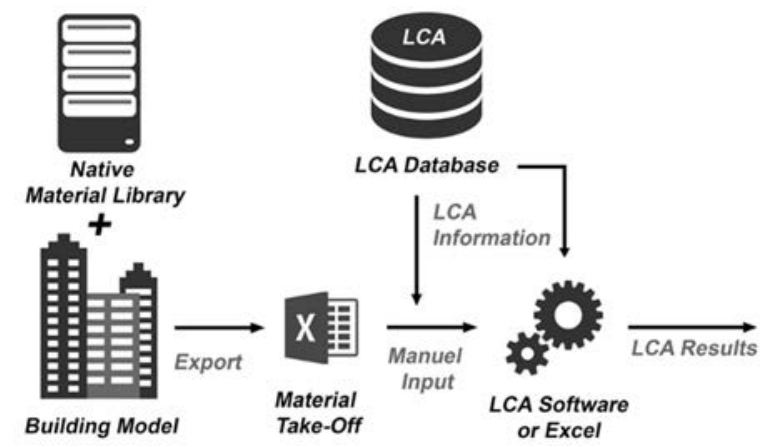

Figure 1: Convectional LCA methodology.

After the material take-off list is generated, there is a need to get the relevant LCA information from a material LCA database to be able to calculate the environmental impact of each component. This calculation can be done in a developed LCA software where the data entry has to be done manually. Usually, licensed software tools already have a link to LCA databases. However, if the LCA calculation has to be done in a spreadsheet-based tool, it might be that both material input and environmental impact information have to be typed in directly into the spreadsheet.

Conventional LCA methodology was the commonly known method to generate LCA results for buildings, but there are some difficulties with this kind of approach, which does not make the LCA acceptable for the building professional in everyday work. For example; if the design software tool does not have a parametric interface with the database behind the material, the take-off has to be done by hand, which can be very challenging where the error rate is also very high. As the existing software tools are usually standalone solutions, there is a need to transfer the data from the platform to another. Having such inefficient and repetitive data entry will cause enormous time consumption. Because of manual data input, there is a high potential for mistakes in information transfer. Human-based errors will cause wrong LCA results in the end, which do not represent the project's real environmental impact. 
Another drawback is that the whole information flow is one way where there is no direct feedback to the building designer. Having too many manual entries during the process and also having a separate LCA software with several settings, obligate the industry to have an expert with a certain level of LCA knowledge in this process to be able to do this environmental impact estimation.

\subsection{Static BIM-LCA approach}

The second LCA methodology is a static approach, where the LCA calculation is done based on a frozen state of the BIM model. Information is directly extracted in a standardised exchange format to allow the LCA tools to make the calculations. This flow of information is one-way only.

Direct access to the information in the BIM model in order to calculate the LCA by using an interoperable IFC (Industry Foundation Classes) format and inserting it in LCA software can be defined as "Static Approach". Static approaches evaluate the entire life cycle of the building, thus achieve a more accurate approach to LCA evaluation compare to conventional methods. As the whole building information is extracted in one format like IFC or gbXML (Green Building XML-Schema), there is no need to enter the building information in the LCA tool again, and this will avoid any manual re-entry. As every extracted data will represent one state of the project, different alternatives can be compared and documented. The results can highlight the hotspots where intervention is required and thus enhance environmental performance. (Fig. 2)

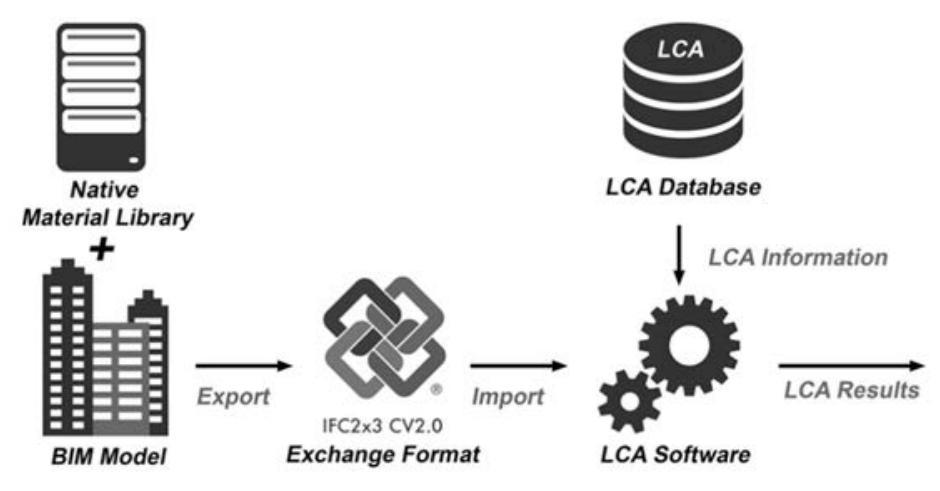

Figure 2: Static approach diagram.

On the other hand, a static approach has some drawbacks. As the performance of the LCA is not developed in the BIM software itself, any changes in the BIM model can only be made by going back to the BIM software and re-importing the model into the LCA platform, which can be very time-consuming [4]. Also, the interoperability between BIM models and LCA tools has not been fully developed yet. The transmission from a BIM platform to LCA platform is done via IFC format into a common database, where it can be combined with the LCA inventory database. The accuracy of the extracted BIM model in a standardised data format is limited and has a narrower range of environmental information, which makes the importing of the file into the LCA software and the calculation less accurate. The challenge is not to lose information during this method. 


\subsection{Dynamic BIM-LCA approach}

The last LCA methodology, which is also the focus of this research, is called the dynamic approach where the environmental impact assessment is done with real-time monitoring during the entire planning process. Environmental properties and all the relevant LCA information are included in the BIM models, objects, and materials. The information flow is bi-directional, which allows a potential optimisation and a feedback loop.

The inclusion of environmental properties in the BIM objects allows the extraction of environmental quantities directly from the model. Such an approach is more comfortable and faster and sets environmental criteria highlighted for decision-making. Nevertheless, it is still necessary to improve the availability of information on construction materials as there are unsolved issues regarding automatically inclusion of information related to transportation, location and regional characteristics of the project [4].

The dynamic approach seeks to find an automatic and efficient link between BIM models and environmental information included in the LCA databases. Therefore, there is a need for an LCA tracking number in the native material library of the design software. Once this material mapping is done, all the material used in the BIM model will be matched with its corresponding real material recognised in the national LCA database. Once the geometry and material information are extracted, with the help of a parametric tool and LCA information coming from LCA database, the environmental impact of each element used in the BIM model can be calculated. This LCA results can be forwarded back to the BIM model in order to create a feedback loop to the designer (Fig. 3). This feedback can allow real-time LCA monitoring, which can be followed by an LCA optimisation directly in the designer's software. As the parametric tool is continuously analysing the LCA results, they can be extracted at any moment as a report for further uses.

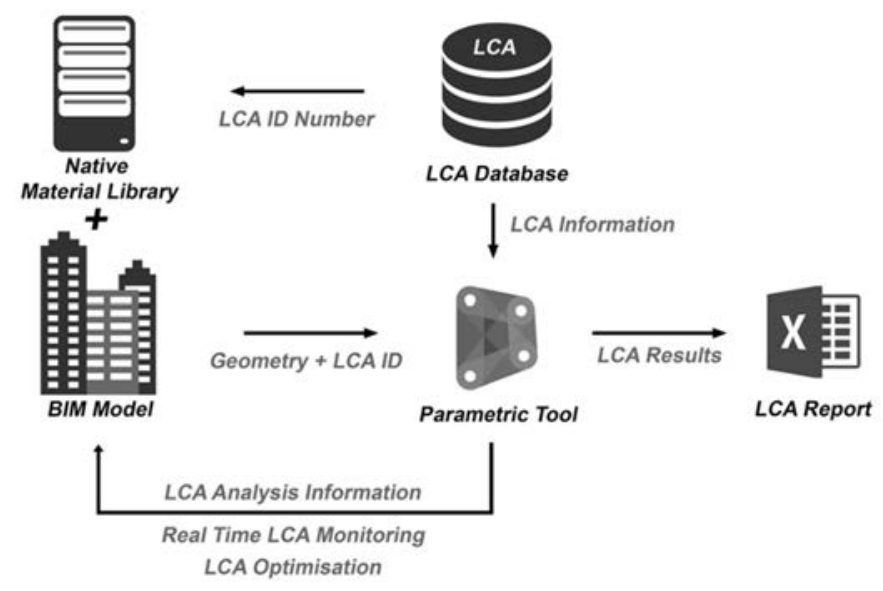

Figure 3: Dynamic approach diagram.

In the dynamic approach, BIM objects have continuously updated environmental properties based on LCA calculations in their properties. In this way, it is possible in the predesign and design phases to include environmental criteria in the decision-making process regarding the choice of materials and building elements. LCA monitoring allows the designer to make an environmental optimisation at any given time directly in design software. In this 
workflow, it is essential that designers can understand the environmental information given and its meaning in order to able to compare between different alternatives. Since any input to the building information model with the dynamic approach will directly result as environmental impact result. It has direct feedback to the BIM model, as the information flow is multi-directional.

Since the BIM-integrated LCA solutions are not that advanced and are lacking an updated link to LCA databases, the LCA results, which will be gathered from a dynamic approach will be less accurate compared to a static approach. Further developments are needed to improve the efficiency of this approach. The LCA of this approach is still material and objectoriented. Also, it does not include the environmental impact caused by the production of the material, transportation, and installation. This information can be included in the BIM model based on different characteristics, location, time and features of each project. However, once the accuracy of BIM models is increased, it can be considered as a prototype for the building, which is going to be realised.

\section{DEVELOPMENT OF DYNAMIC LCA TOOL FOR BIM}

Revit supports creating BIM models, which are object-based data models containing relevant information of each object. There are several parametric LCA tools, which help to assess the environmental sustainability of buildings. Every visual programming tool requires several inputs to generate results [7]. The aim is to keep the same configuration of the previously developed LCA tool and investigate the requirements to integrate it into any BIM platform. To be able to do this, the input data should come from one BIM model where every included data behind should be carefully understood [8].

In this study for the LCA calculation, the relevant geometrical information like material, volume, area is taken from the BIM Model hosted in the Revit platform. The necessary environmental impact information is extracted from the swiss material-based life cycle assessment database from KBOB (Coordination conference of the building and real estate authorities of public clients, where KBOB in German stands for "Koordinationskonferenz der Bau- und Liegenschaftsorgane der öffentlichen Bauherren"). This link is done with the help of visual programming tool called Dynamo, which is running directly over the Revit interface as a plugin.

In order to develop an LCA tool in Dynamo, which can read LCA information directly from a building model via a link to a database with environmental data and reference service life (RSL) data of the components is needed. Dynamo/Revit can calculate the mass of the original materials, which is used to do the multiplication for LCA information. Every LCA result can be written back to every single element object in the BIM model.

\subsection{Concept: dynamic LCA with BIM}

Among LCA-BIM methodologies, a dynamic approach has the most promising possibility to have direct feedback to the building professional at the exact time where the design decision is taken. For the dynamic approach, the critical thing is to have a material mapping between LCA database and native material library. This mapping can be done with one standard LCA ID number, which matches on both platforms. As the material in the native material library will be able to get the material properties and environmental impact values from the LCA database. Together with the geometrical information from the Revit BIM model and LCA information from the database, Dynamo can combine and calculate all the LCA results directly from each building element in the BIM model. The LCA results can be extracted as an LCA report for reporting purposes of discrete states and be transmitted to each BIM 
element properties directly. The environmental impact information of each element will allow the designers to have a continuous monitoring capability. Also, by illustrating these results, designers can optimise the building directly in the design software.

\subsection{Material mapping for LCA-BIM}

The material mapping between the design tool and the international LCA database is one of the primary keys for successful LCA-BIM integration [9]. As the material names may vary in each project because of language differences, regional or local definition, creating a namebased mapping is not the optimal solution. Therefore, there is a need for a general material identification that can match differently named but the same materials. This identification can be made with a common LCA ID number. If this number is successfully distributed to the native material library, it is also possible to match the necessary LCA information to the element. The biggest challenge in a material mapping is that it must be done manually. This way, the potential incorrect matching due to wrong name inputs leading to inaccurate LCA results still exists. As it can be seen in, for the same material, the names from LCA databank to native material database can vary a lot.

Table 1: Example of material mapping from KBOB to Revit native material library.

\begin{tabular}{|c|c|c|}
\hline KBOB ID & Material name in KBOB Database & Material name in Native (Revit) \\
\hline 01.014 & Armierter Beton (HB, CEMII/B) & GHA_Beton Typ BE2 \\
\hline
\end{tabular}

\subsection{LCA parameters in Revit}

Next to using the BIM data together with the LCA database and generate LCA results, one of the primary goals is writing all the calculated values back to each element in the design software. In order to do so, there was a need to define specific parameters that are used for the calculated LCA values on the element level.

The first parameter of an element ID, LCA Category, is used for the identification of each element. The indicators Global Warming Potential (GWP), Eco-points (UBP - based on the Swiss method of ecological scarcity), and primary energy non-renewable (PEF-ne) from the KBOB database are used and represent the total environmental impact of all BIM element. The floor area-based values for PEnr $\left(\mathrm{MJ} / \mathrm{m}^{2} \cdot \mathrm{a}\right)$, Minergie index $\left(\mathrm{kWh} / \mathrm{m}^{2} \cdot \mathrm{a}\right)$ and GWP $\left(\mathrm{kg} \mathrm{CO}_{2}-\mathrm{eq} / \mathrm{m}^{2} \cdot \mathrm{a}\right)$ are showing the ratio of total environmental impact to area and year in order to be able to make a comparison with other building elements.

For the LCA calculation, there is a need for information that is valid for the complete BIM model such as reference service life, gross and heated floor area, as well as the model version to separate different results from each other. All this information is written under the global parameters.

\section{DYNAMO SCRIPT MODULES}

Dynamo is a visual programming plugin for the Revit design software, which creates a direct link to the building information model data and allows to make some processing based on the scripts. In order to make LCA calculation based on the BIM model and KBOB database, there was a need to link them logically. Depending on the project size, there might be thousands of element objects that have to be calculated and the results written back to every single element. This kind of process might require too much computing power, and therefore, there is a need to split the environmental impact assessment into logical work packages. 
These scripts need to be run one after another to have a successful LCA calculation. There are modules for model preparation, for Structural/Envelope/Interior Elements, for building system and finally modules for LCA optimisation and result modules.

Table 2: Dynamo script module overview.

\begin{tabular}{|l|l|}
\hline \multirow{5}{*}{ Preparation } & $\begin{array}{l}\text { M0_Material List Fill: Based on a pre-trained material mapping } \\
\text { spreadsheet the missing KBOB numbers are filled in the native material } \\
\text { library }\end{array}$ \\
\cline { 2 - 3 } & $\begin{array}{l}\text { M1_Create Project Parameter: LCA parameters for each element as well } \\
\text { as the global parameters are generated }\end{array}$ \\
\cline { 2 - 3 } & $\begin{array}{l}\text { M2_Fill Global Parameter: General information about the project model } \\
\text { to the global parameters are added }\end{array}$ \\
\hline \multirow{5}{*}{ elemstruction } & $\begin{array}{l}\text { M3_Wall: Analyse all wall elements and calculate the environmental } \\
\text { impact }\end{array}$ \\
\cline { 2 - 3 } & $\begin{array}{l}\text { M4_Floor: Analyse all floor elements and calculate the environmental } \\
\text { impact }\end{array}$ \\
\cline { 2 - 3 } & $\begin{array}{l}\text { M5_Structural: Analyse all structural elements and calculate the } \\
\text { environmental impact }\end{array}$ \\
\cline { 2 - 3 } Technical & $\begin{array}{l}\text { M6_Envelope: Analyse all external and facade elements and calculate } \\
\text { environmental impact }\end{array}$ \\
\cline { 2 - 3 } elements & $\begin{array}{l}\text { M7_Interior: Analyse all interior elements and calculate environmental } \\
\text { impact }\end{array}$ \\
\hline \multirow{5}{*}{ Results } & $\begin{array}{l}\text { M8_Technical Routing: Analyse all technical routing elements like pipe, } \\
\text { ducts, cable trays and calculate the environmental impact }\end{array}$ \\
\cline { 2 - 3 } & $\begin{array}{l}\text { M9_Technical Devices: Analyse all technical device like lightings, } \\
\text { equipment, plumbing fixtures and calculate the environmental impact }\end{array}$ \\
\hline $\begin{array}{l}\text { M10_View Duplicate: Duplicate the open 3D view to illustrate the LCA } \\
\text { Results }\end{array}$ \\
\cline { 2 - 3 } $\begin{array}{l}\text { M11_Visualisation: Color the elements in the duplicated view to } \\
\text { illustrate the ones are above or below limit values }\end{array}$ \\
\cline { 2 - 3 } $\begin{array}{l}\text { M12_LCA Report: Generate a summary report from the LCA of the } \\
\text { BIM model and export to a spreadsheet }\end{array}$ \\
\hline
\end{tabular}

\subsection{Preparation modules}

Before any dynamic LCA calculation, there is some pre-work that has to be completed. As new materials might be added to the native material library in ongoing projects, there should be one script that assigns the material ID to the materials and makes sure that all the materials are taken into consideration. Another script should assign all the required parameters to the BIM objects to be able to fill later on the environmental assessment results. Finally, the global parameter containing general information about the project, such as assessment year or total floor area has to be added. After running Module 0 to Module 2, all the parameters are filled, and the native material library is updated so that the dynamic environmental assessment can proceed. 


\subsection{Construction elements modules}

Module 3 to Module 7 have a similar logic of their algorithms. First, they extract the containing materials for each element and then their volume and area information respectively to those elements. Breaking down each building element to its composition allows to extract the material units from each element and do the LCA calculation accordingly. Every material unit is matched again with its elements and based on the element properties; the LCA categorisation can be done. Finally, all Revit elements' LCA parameters are filled with the calculated LCA result values (Fig. 4).

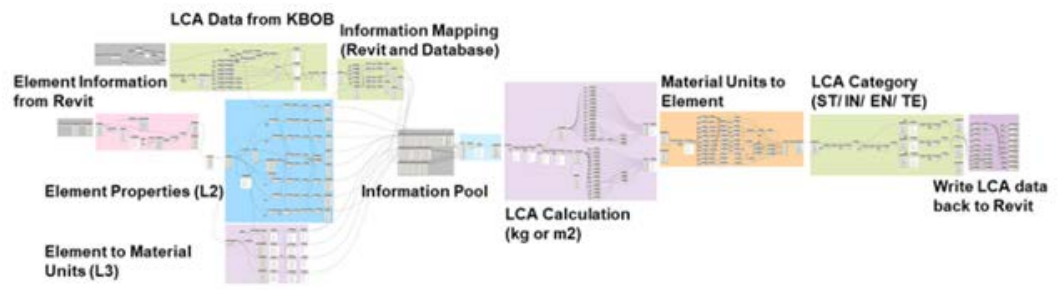

Figure 4: Dynamo script module 3 - walls (example for structural/envelope/interior elements LCA calculation).

\subsection{Technical elements modules}

Technical elements are slightly different from other building elements in the model. Technical elements can be grouped into two subgroups, which are technical routing and technical devices. Technical routing elements are the pipes, ducts, cable trays and their fittings, i.e. elements that are generated by extruding a cross-section through a line path.

Table 3: Dynamo script concept for technical elements.

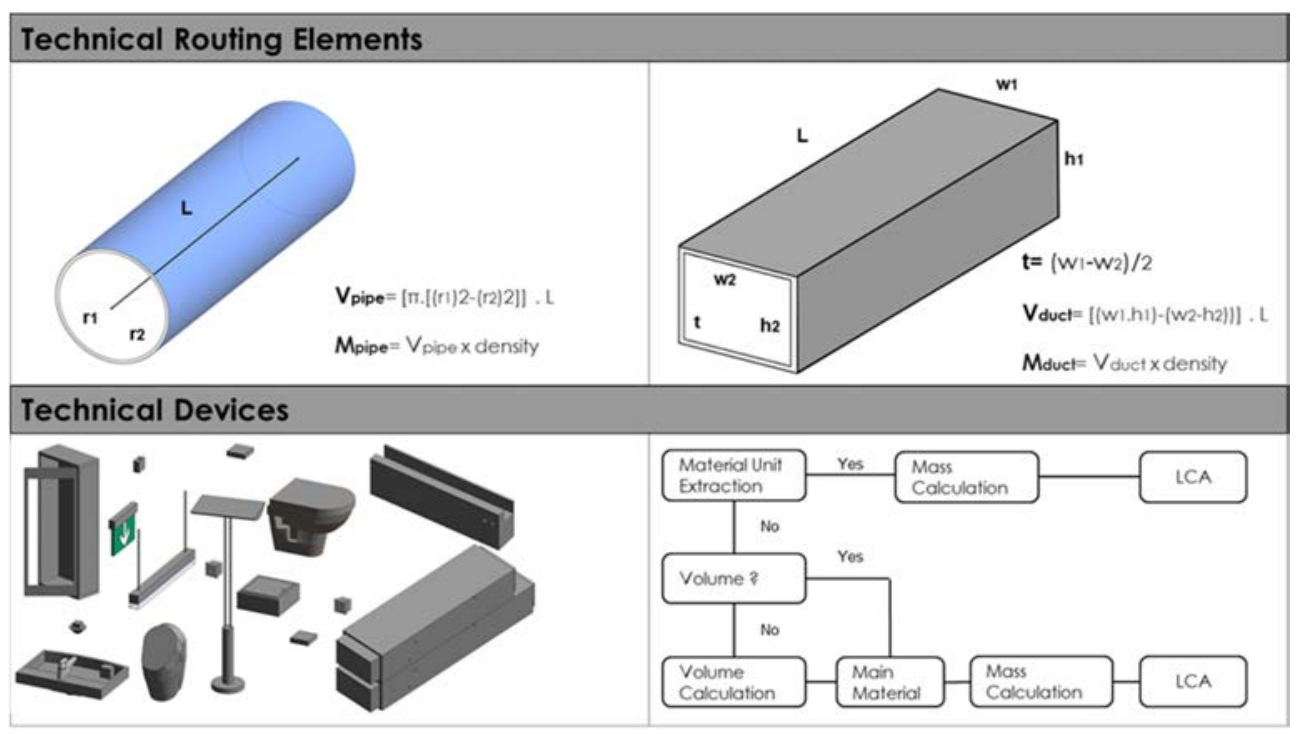


Technical routing elements represent $1 / 3$ of the whole BIM model element quantity, which might cause in a complex project performance problem to the design software. To overcome the performance issue, as such that technical routing elements do not have a high level of geometry, but they are there for a better level of information. Dynamo cannot access directly to the volumetric information of these elements, which makes the material mass of components calculation more difficult. Therefore, a straightforward solution is to calculate each cross section and multiply it with the reference line length. The necessary information to calculate the area from the cross section was written as information in these elements. Based on this information, it is feasible to generate the volume and correspondingly the mass value for each element, which allows calculating the LCA impact afterwards.

Technical devices consist of all the products that are bought and installed in the building where usually the dimensions and contents are fixed by the producers. These elements are categorised in Revit as duct and pipe accessories, air terminals, lighting fixtures, electrical and mechanical equipment, data services and plumbing fixtures.

In building information models, the geometry is usually simplified for performance reasons and used as a placeholder. Ideally, their producers know how much environmental impact is caused by each of their product, and it would be easier to make the LCA calculations and provide the information. Therefore, it is essential that the producers provide the material content of their products and if possible, directly also the environmental impact included as information to the element.

\subsection{Results modules}

After running all the modules from 0 to 9, all elements' LCA parameters are filled with the corresponding calculated environmental impact values. One way to illustrate the results is by providing a colour code on the $3 \mathrm{D}$ model to the building professional to indicate the possibilities for optimisation. Another way is to extract all the information as a summary in a report. The dynamo script module 12 extracts a total value for four LCA categories (Structural, Envelope, Interior, Technical) and as well as total value for each Revit category. The LCA report allows monitoring the LCA development for that stage of the design process.

The overall LCA results for each category can also be illustrated graphically in order to point out the element groups, which have the highest environmental impact. The report provides a model version and execution time, which allows comparing different data in a continuous planning process.

\section{CASE PROJECT}

After developing the method to realise the BIM-LCA integration through Dynamo, the tool is used in a real case project. In this study, the design process of a real case study building is analysed. The case study is the three-storey extension of an office building of the engineering company Basler \& Hofmann in Esslingen, Switzerland. The project was a BIM pilot to test the building process only based on the digital model without printed 2D plans.

The design process of a building is continuous, and correspondingly the BIM model evolves continuously. To be able to track the development of the BIM model, the current state as a sample model was analysed. The building permit was taken in the mid-December 2018. Until then, the LOD (Level of Development) of the model was low. Therefore, it was sufficient to get a sample from each month in the building permit stage until December 2017. From January 2018 on a sample was taken each week, which resulted in a sample of 34 models. 
Fig. 5 presents a preliminary but informative overview of the LCA development process of the design. The most striking feature is that, as the design develops further down, the new entities and placeholder objects are replaced with more specifically designated identifiers. These changes in the curve based on the time axis leads to a much refined and desired design solution designated with the region of the settled curves. Another importing thing to notice in this graph is the environmental impact of all categories reach an absolute constant after week 18, and there are only slight changes afterwards.

Another advantage of gathering all the LCA data from each element in a BIM model allows focusing on specific categories. Fig. 6 represents the environmental impact development through design processes for all structural, interior and envelope element categories.

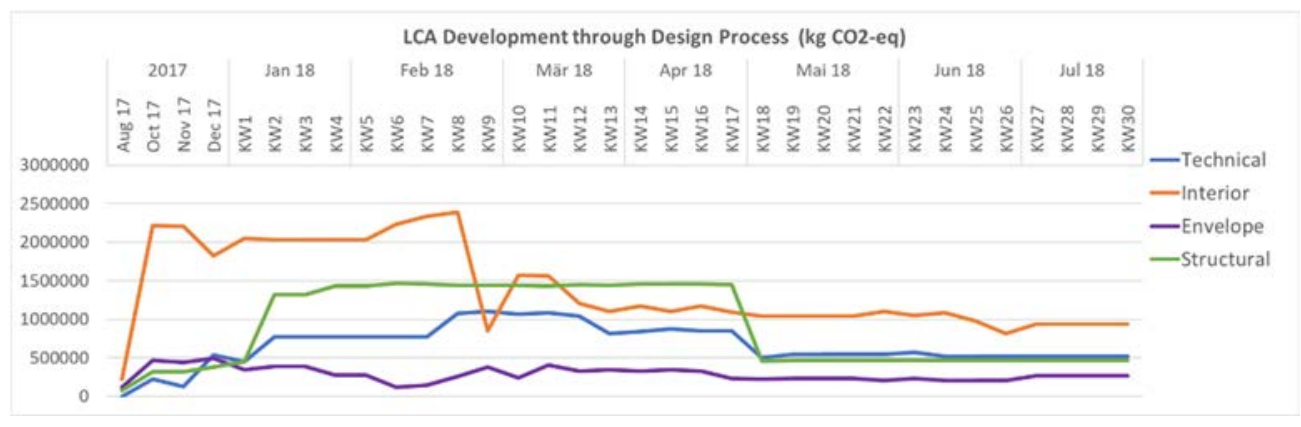

Figure 5: LCA development through the design process.

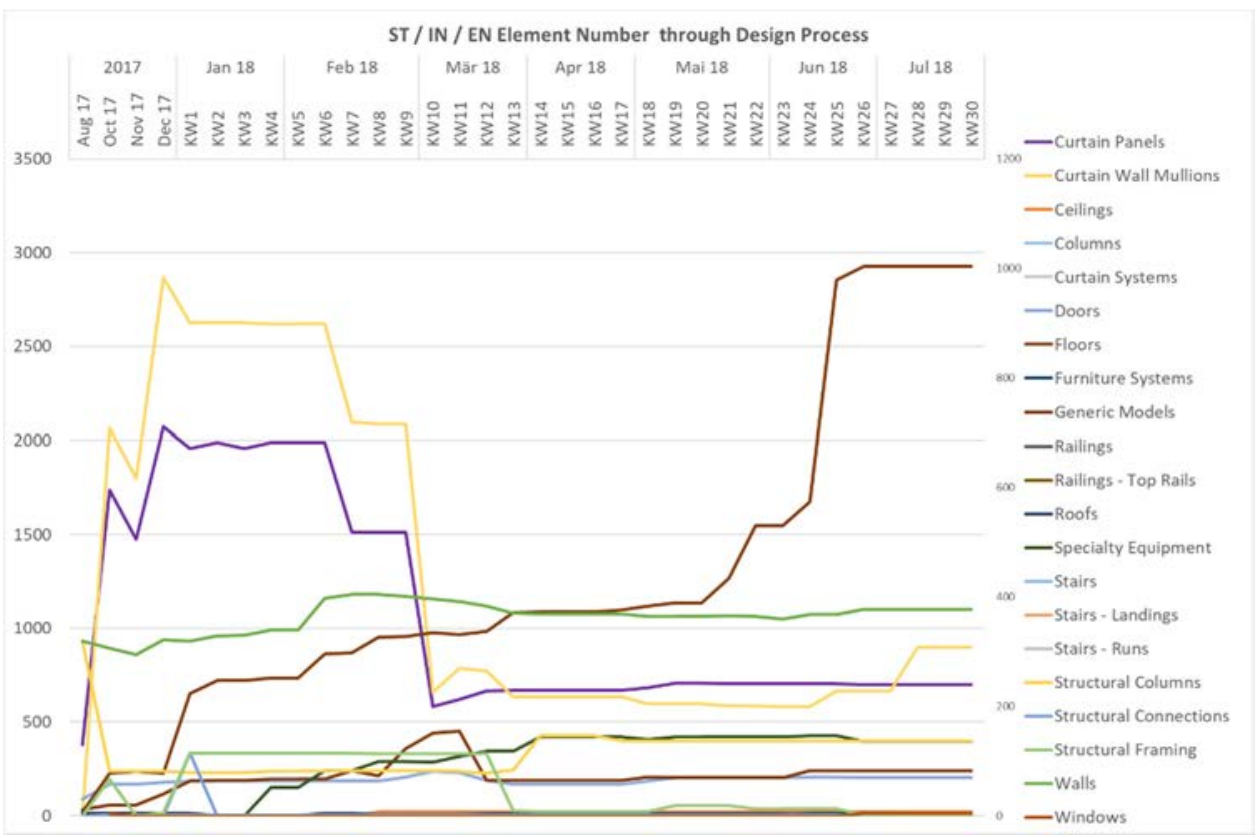

Figure 6: Structural, interior, and envelope element number through the design process. 


\section{CONCLUSION}

BIM-LCA integration has enormous potential for future sustainable development of the construction industry. Using all available building information and linking them with existing LCA databases has the potential to illustrate the environmental impact of the decisions being taken by building professionals. Any LCA result during the decision process might lead to sustainable development in the early stage of the planning, where the changes are still affordable.

This study aimed to realise a fully automated BIM-LCA integration in order to reduce the effort of LCA, to increase the acceptance, to eliminate the need of particular LCA expertise, to create a shared understanding, and to develop a real-time LCA monitoring during all design phases to continuously influence the decision-making process by building professionals. To reach this goal, a BIM-integrated, and fully automated LCA tool was developed in order to test it on a real case planning process to see the limits and potentials.

A BIM-based dynamic LCA with a parametric tool throughout the design process has a lot of added value to the building professionals in order to reduce the embodied energy from their design. First, having a fully automated LCA tool will scale down the effort to generate environmental impact results and save time. The integrated dynamic approach will also reduce the cost of carrying out LCA, which will increase the acceptance from the construction industry. BIM-LCA integration will allow illustrating LCA results directly in the design software environment. Thanks to this integration, environmental aspects will get a parallel workflow to the existing planning process. Integrating LCA into a regular planning process will escalate the acceptance and understanding of sustainable planning. This feature will also help to cut down on the individual need of LCA expertise from every building professional to be able to evaluate the building regarding sustainability. Another advantage of parametrising the LCA methodology based on BIM is to diminish the human error mistake by preventing the repetitive data re-entry, which will increase the reliability of LCA results.

Merging BIM and LCA methodology shifts the understanding from having a component level environmental impact assessment to the element level. Regarding optimisation, this will allow having a better overview of environmental impacts on the element level. Finally, the real-time LCA monitoring over the whole building for each element will potentially influence the material choice and the design decision process at the exact time where the decisions are taken.

As the public awareness of the consumption of energy and resources increases, BIM and LCA integration becomes a necessity to promote environmental assessments during the standard planning processes.

To sum up, the necessity to integrate LCA and BIM in the decision-making process at an initial stage cannot be ignored in the future. The methodological challenges and limitations of existing LCA-BIM tools show that there is still an immense research potential for the development of new evaluation tools and methodologies. Once the integration has been successfully done, thanks to information-based building modelling, the LCA will take part in all stages of construction planning and will be done continuously in real time.

\section{REFERENCES}

[1] Kwok-Wai Wong, J. \& Kuan, K-L., Implementing 'BEAM Plus' for BIM-based sustainability analysis. Automation in Construction, 44, pp. 163-175, 2014.

[2] Bueno, C. \& Fabricio, M.M., Methodological discussion of insertion and exportation of LCA data embedded in BIM elements. WIT Transactions on the Built Environment, vol. 169, WIT Press: Southampton and Boston, pp. 101-110, 2017. 
56 Building Information Modelling (BIM) in Design, Construction and Operations III

[3] The Strategy for Sustainable Construction, HM Government, Department for Business, Enterprise \& Regulatory Reform, Construction Sector Unit. https://webarchive.nationalarchives.gov.uk/+/http:/www.bis.gov.uk/files/file46535.p df. Accessed on: 20 Aug. 2018.

[4] Díaz, J. \& Anton, L.Á., Sustainable construction approach through integration of LCA and BIM tools. Computing in Civil and Building Engineering, ASCE 2014 Conference, pp. 283-290, 2014.

[5] Soust-Verdaguer, B., Liatas, C. \& García-Martínez, A., Critical review of BIM-based LCA method to buildings. Energy and Buildings, 136, pp. 110-120, 2017.

[6] European Commission: Institute for Environment and Sustainability, ILCD Handbook: Analysis of Existing Environmental Impact Assessment Methodologies for use in Life Cycle Assessment, 1st ed. https://eplca.jrc.ec.europa.eu/uploads/ILCDHandbook-LCIA-Background-analysis-online-12March2010.pdf. Accessed on: 17 Aug. 2018.

[7] Hollberg, A., A Parametric Method for Building Design Optimisation Based on Life Cycle Assessment, Bauhaus-Universität: Weimar, 2016

[8] Cavalliere, C., Dell'Osso, G.R. \& Pierucci, A., Life cycle assessment data structure for building information modelling. Cleaner Production, 199, pp. 193-204, 2018.

[9] Hollberg, A., Parametric Life Cycle Assessment: Introducing a Time-Efficient Method for Environmental Building Design Optimisation, 1st ed., Bauhaus-Universität: Weimar, 2017. 